\title{
Effets maîtres, effets établissements: quelle responsabilité pour l'école?
}

\section{Marie Duru-Bellat}

La diversité des situations éducatives et des contextes scolaires est volontiers considérée en France comme une des facettes importantes de la crise de l'école. Mais ne faut-ily voir que la traduction sur le terrain scolaire de la ségrégation sociale de l'habitat? Ou bien faut-il admettre que l'école elle-même participe à la définition de ces situations éducatives contrastées?

Ce texte présente une synthèse actualisée des recherches françaises consacrées aux effets de contexte - effet maître ou effet établissement - en insistant sur les problèmes méthodologiques qu'ils posent, sur leurs nuances et leur complexité (ces résultats étant eux-mêmes contextualisés), sur la nécessité de distinguer efficacité et équité. Il souligne également les controverses qui les marquent, et, pour finir, leur portée politique, qu'il s'agisse de la mobilisation des enseignants ou du "contrôle» des demandes parentales, mais aussi, et plus fondamentalement, de la capacité de l'institution scolaire à offrir à tous un environnement éducatif d'égale qualité.

Le constat de la diversité des situations éducatives et des contextes scolaires est devenu extrêmement répandu en France, à tel point que l'accroissement des écarts entre établissements serait aujourd'hui un des symptômes importants de la crise de l'école. Effectivement, tout semble opposer un collège de banlieue populaire et un collège "chic» de centre ville. Mais ces contrastes ne sont-ils que le reflet, la traduction sur le terrain scolaire, de la ségrégation sociale de l'habitat? L'école, dans ce cas, hériterait de situations plus ou moins difficiles à gérer, et elle s'y attellerait au mieux, avec les moyens dont elle dispose. Mais on ne peut refuser d'envisager que l'école elle-même participe à la définition de ces situations éducatives contrastées: quand, après plusieurs années de fréquentation scolaire, des écarts sensibles opposent les performances en lecture d'enfants d'écoles différentes, peut-on véritablement exclure que cette fréquentation scolaire n'ait aucune responsabilité en la matière? Cette question est tout sauf anodine: démontrer que selon le maître qu'il a ou l'école qu'il fréquente, un enfant a des chances très différentes de progresser, c'est reconnaître que tout n'est pas joué en fonction des atouts ou des handicaps dont il aurait hérité, c'est mettre à jour un des «fac- 
teurs de production» de l'échec scolaire. C'est aussi interpeller directement l'institution scolaire dans sa responsabilité.

En France, les recherches sur les effets de contexte (effet maître ou effet établissement) se sont développées à partir des années 80 , assez tardivement par rapport aux pays anglo-saxons. Ceci pour des raisons sans doute à la fois théoriques, institutionnelles et méthodologiques. Théoriques, car le poids du paradigme de la reproduction par l'institution scolaire des inégalités sociales a longtemps empêché de penser les marges de manoeuvre pouvant exister dans le quotidien des classes et des établissements. Institutionnelles, car le fait même que le système soit centralisé de droit rendait inconcevable qu'il puisse de fait fonctionner de manière différente d'un site à l'autre. Méthodologiques enfin, car rien n'est moins immédiat que d'appréhender des effets de contexte, et longtemps les chercheurs français n'ont maîtrisé que des instruments statistiques trop frustes pour appréhender la notion de valeur ajoutée, fondement même des effets de contexte.

\section{Des différences entre contextes scolaires à la notion d'effet contextuel}

Parmi les informations couramment disponibles, des données statistiques abondantes attestent d'écarts sensibles dans les "performances» du système éducatif d'un lieu à l'autre, qu'il s'agisse des taux d'obtention du baccalauréat par lycée, des taux de passage ou de redoublement par collège, des scores à des épreuves communes de connaissance d'une classe à l'autre... Ces écarts sont un point de départ et laissent place pour des interprétations variées: dira-t-on que tel maître ou telle école est particulièrement inefficace ou que s'y trouvent réunies les conditions les pires pour un enseignement valable? On se doute bien, mais encore faut-il l'établir, que les performances d'un établissement ne seront pas les mêmes selon qu'il accueille moins de 1\% d'élèves en retard - c'est le cas de 10\% des collèges français -, ou au contraire plus de $10 \%$ - c'est aussi le cas de $10 \%$ des collèges -, ou encore selon qu'il accueille moins de $20 \%$ d'élèves de milieu favorisé - comme $10 \%$ des collèges - ou plus de $64 \%$ - 10\% des collèges sont dans cette situation ...

Pour éclairer cette question, des analyses statistiques multivariées sont nécessaires, incorporant non seulement, comme leur nom l'indique, plusieurs variables - caractérisant les élèves, d'une part, les contextes en présence, d'autre part -, mais aussi des observations collectées à deux moments du temps - niveau initial des élèves, niveau final -, puisque si efficacité de l'école il y a, elle prend nécessairement place dans un laps de temps donné. En d'autres termes, et c'est là la notion de valeur ajoutée, ce qu'il faut évaluer, c'est ce que des élèves initialement comparables (même caractéristiques sociales, même niveau scolaire...) «gagnent» ou «perdent» spécifiquement quand ils sont scolarisés dans tel ou tel contexte. 
Ceci suppose un travail en trois étapes: l'observation des effets bruts d'établissements tout d'abord, sur la base des scores scolaires obtenus en fin d'année dans les différents sites étudiés; puis l'appréhension d'effets nets c'est à dire le plus ou le moins en termes de progression associé à la fréquentation d'un établissement pour des élèves ayant un niveau initial identique et des caractéristiques socio-démographiques comparables. A ce stade, les effets établissements sont encore des boites noires, mais la différence entre effets bruts et effets nets n'en est pas moins instructive: tel établissement a peut-être des scores bruts élevés tout en faisant peu progresser des élèves au départ déjà bons ou de milieu aisé; cet écart révèle les effets de composition résultant de l'agrégation, dans tel ou tel établissement, d'élèves dotés de telle ou telle caractéristique pertinente par rapport à la réussite. Il reste, dans un troisième temps, à s'efforcer d'expliquer ces effets nets en remplaçant la variable établissement par un certain nombre de ses caractéristiques objectives (composition sociale du public, structure du corps enseignant, offre de places...), ou encore, de manière plus qualitative (mais cette voie est peu pratiquée), par certains traits de son fonctionnement interne.

C'est à ce stade que sont mis en évidence d'éventuels effets contextuels, résultant de la concentration de telle ou telle variable individuelle ou environnementale, au-delà des effets de composition; par exemple, pour des élèves de milieu populaire, le seul fait d'être scolarisé dans un collège "chic» (dont le pourcentage d'élèves de milieu populaire est faible) a-t-il une importance sur les progressions qu'ils réalisent, ou réciproquement, les élèves de milieu aisé "perdent»-ils à fréquenter des établissements populaires? Il reste qu'au-delà de ces effets contextuels qui ont un caractère général (ils marquent le fonctionnement des collèges «chics» ou des collèges populaires en général), il y a place pour des effets singuliers d'établissement, relevant de leur politique par exemple, qu'il faudra ensuite expliquer. Mais si cette dernière composante correspond assez bien à la notion commune d'effet établissement, il faut souligner qu'elle ne peut être véritablement appréhendée qu'au terme d'un processus d'investigation structuré et relativement long. Il faut tout d'abord collecter des données individuelles nombreuses, dans des contextes le plus varié possible, informations ensuite soumises à une exploitation statistique modélisée. Il faut également construire des mesures des acquis des élèves relativement indépendantes du contexte et notamment de l'établissement fréquenté, ce qui exige l'élaboration et la passation d'épreuves communes standardisées. ${ }^{1}$

En France, ce sont les économistes qui ont les premiers utilisé des approches de type valeur ajoutée dans le domaine de l'éducation (avec les travaux de l'IREDU dans les années 80 ), alors que dans les pays voisins, ce type d'approche faisait «normalement» partie des sciences de l'éducation, avec le courant dit du school effectiveness, très développé en particulier aux Pays-Bas ou au «RoyaumeUni. C'est ainsi qu'en 1984 paraissait le premier article estimant l'«effet maître» en primaire (Mingat, 1984), et en 1988 le premier article évaluant les effets établissement au niveau du collège (Duru-Bellat $\&$ Mingat, 1988). Ces premières es- 
timations lançaient en fait tout un programme de recherche, puisqu'elles ne faisaient que "pointer"» des effets contextuels sans s'engager autrement qu'à titre exploratoire sur les caractéristiques du contexte pertinentes à cet égard. Ce courant a depuis été développé par des chercheurs en sciences de l'éducation comme Bressoux (1994 et 1995) ou Grisay (1993 et 1997), mais aussi, récemment, par des sociologues comme Felouzis (1997) ou Cousin (1998). Se sont également développées, notamment à l'INRP avec les travaux de Derouet (1992), des approches plus descriptives des contextes scolaires, fondées sur des observations ethnographiques, qui s'intéressent moins aux effets des caractéristiques du contexte sur les élèves qu'aux logiques qui organisent l'identité des établissements et régissent le fonctionnement des acteurs enseignants. ${ }^{2}$

\section{Des établissements inégalement performants}

L'ampleur des effets école est bien entendu contextualisée: selon les pays, les écoles peuvent se différencier plus ou moins. En France, les estimations faites par Grisay (1997), au niveau du collège, révèlent des effets établissement assez modérés, par rapport aux pays voisins: comparant les progressions de collégiens au départ semblables, ayant effectué leur scolarité dans des établissements différents, Grisay estime que $5 \%$ de la variance du niveau final en mathématiques (un chiffre un peu plus faible étant obtenu pour le français) s'explique par le collège fréquenté (les recherches anglaises ou néerlandaises débouchent sur des chiffres se situant plutôt entre 6 et $8 \%$ ). Cet effet, si faible qu'il apparaisse de prime abord, signifie tout de même que les élèves scolarisés dans l'établissement le plus efficace obtiennent au sortir du collège un score en mathématiques supérieur d'un écarttype à celui qu'atteignent des élèves qui, à l'entrée, étaient de même niveau mais ont eu la malchance de fréquenter le collège le moins efficace. Ou encore que la position d'un élève obtenant dans son collège un score moyen (le situant au 50e rang sur 100) peut varier, selon l'établissement fréquenté, du 33e au 67e rang par rapport à l'ensemble de la population.

$\mathrm{Au}$ niveau primaire, une vaste enquête sur les acquis en lecture (Bressoux, 1995) a montré à nouveau un effet école relativement faible en France ${ }^{3}$, par rapport aux pays voisins: un peu moins de $5 \%$ de la variance des scores au terme de la seconde année de primaire s'explique par l'effet école, alors que durant cette même année, les caractéristiques personnelles de l'élève (origine sociale, âge, notamment) ne pèsent que pour environ $4 \%$ de la variance, le tout à caractéristiques initiales données bien sûr. C'est dire que sur les progressions qui se jouent pendant une année, l'école fréquentée pèse plus que l'origine sociale, même si, sur l'ensemble de la carrière scolaire, l'origine sociale va cumuler ses effets de manière plus systématique qu'en ce qui concerne l'école ou le maître. Notons que ces effets école sont plus marqués chez les élèves les plus faibles (ils sont deux fois plus forts que pour la moyenne des élèves), alors qu'à l'inverse, ils sont de 
peu d'importance sur les élèves les plus forts, dont les progressions sont peu affectées par le contexte.

Une question importante est celle de l'articulation entre ces effets école et les inégalités sociales. La perspective dominante aujourd'hui en France revient à expliquer l'avantage scolaire des enfants de milieu aisé par les atouts qu'ils tirent de leur environnement familial (qualité du suivi scolaire, proximité avec la culture scolaire, type de rapport au savoir, etc.). Dans ce cas, l'inégalité des héritages sociaux s'impose à l'école. Or les travaux de Grisay (1997) amènent à relativiser cette perspective; ils montrent que les élèves de milieu favorisé sont aussi plus nombreux à fréquenter les collèges les plus efficaces, et à bénéficier, de manière générale, des meilleures conditions d'enseignement. Et cette seconde source d'inégalité sociale est loin d'être négligeable: entre collèges défavorisés et collèges favorisés, les différences sont moins grandes eu égard aux caractéristiques du public d'élèves (concernant le suivi parental, l'absentéisme, l'importance que les élèves accordent aux études, le temps passé au travail à la maison...) qu'entre les facteurs propres à l'établissement (discipline, exposition à l'apprentissage, temps perdu en classe...). C'est dire que les établissements sont plus inégaux que les publics qu'ils accueillent, et cette inégalité dans les fonctionnements et les ressources scolaires ${ }^{4}$ se conjugue avec les inégalités sociales dans les ressources familiales que les élèves apportent en quelque sorte avec eux.

Notons enfin que si ce sont les «effets établissement» sur les acquisitions des élèves qui ont été le plus étudiés, cela n'exclut pas l'existence d'effets sur les attitudes civiques ou la sociabilité. Les travaux de Grisay en attestent, montrant aussi qu'il est très rare qu'un collège soit efficace à la fois en termes d'apprentissage, d'attitudes scolaires et d'attitudes socioaffectives. On a également estimé des effets collège significatifs sur le bien-être des élèves, de même ordre de grandeur que pour les acquisitions en français ou en mathématiques (Meuret \& Marivain, 1997). Au total, rien ne confine les recherches sur l'efficacité des établissements à une polarisation sur les seuls acquis académiques, comme l'insinuent certaines critiques de ce qui serait un modèle obsédé par la performance, ignorant que dans certains établissements d'autres logiques peuvent être à l'oeuvre, valorisant davantage l'intégration des élèves ou la convivialité (cf notamment Derouet, 1992). En fait, l'efficacité que les recherches s'efforcent d'évaluer est relative aux objectifs poursuivis; il s'agit simplement d'apprécier les effets de la fréquentation scolaire et des actions pédagogiques entreprises, ce qui donne à cette entreprise une visée très générale, sans doute centrale dans les sciences de l'éducation et en aucun cas réductible à une approche strictement économique, même si, de fait, la complexité des méthodologies requises font que ce sont surtout les économistes de l'éducation qui, en France du moins, la mettent en oeuvre. 


\section{Les établissements efficaces et la chimère d'un portrait type}

La recherche donne également une idée assez précise de ce à quoi ressemble un collège ou une école efficaces (il est plus difficile, nous y reviendrons, de trancher sur ce qui fait qu'un établissement est plus performant qu'un autre). Tout d'abord, les établissements performants sont plus souvent, en moyenne, ceux qui accueillent un public sans problème. On sait depuis le fameux rapport Coleman (publié en 1966) que tous les élèves, notamment les plus faibles, "gagnent» à fréquenter une école au public plutôt favorisé. Ce type de résultat a été retrouvé en France: à la fois les progressions et les orientations des élèves de collège apparaissent plus favorables dans les collèges fréquentés par des élèves de classe moyenne ou aisée. Des études qualitatives (cf. par exemple Thrupp, 1999; Meuret, 1995) éclairent les voies par lesquelles se joue cet effet de la tonalité sociale du public (désigné par le terme school mix dans la littérature anglo-saxonne). Dans les écoles favorisées, la culture des élèves serait majoritairement favorable aux études, les normes de conduite seraient plus proches des exigences du métier d'élève, les enseignants auraient un niveau d'exigence plus élevé, les programmes seraient mieux couverts, etc. Une partie de l'efficacité des écoles est donc en quelque sorte «apportée» par les élèves, en tout cas largement dépendante du school mix.

Mais les facteurs pédagogiques propres à l'école sont tout aussi importants; ceux qui s'avèrent associés à une bonne efficacité (cf. Grisay, 1997) sont assez conformes, en France, à ce qu'on observe dans la plupart des autres pays. Il faut citer en premier lieu une forte "exposition à l'apprentissage», passant par une utilisation optimale du temps scolaire, avec peu de temps perdu, notamment pour la gestion de la discipline. Sont également en jeu des attentes élevées de la part des enseignants, avec à la clé un souci constant d'évaluation. Jouent également positivement la qualité des relations entre enseignants et élèves, ou de la vie au collège, la clarté des règles, l'existence de droits et de responsabilités pour les élèves, un climat paisible. On trouve donc, dans cet ensemble de facteurs «favorables» à la fois certaines caractéristiques d'une pédagogie plutôt «traditionnelle» valorisant le travail et la discipline, et d'autres davantage liées à une pédagogie humaniste (traiter les élèves avec justice, leur accorder des droits...). Par contre, certains facteurs présentés comme le fer de lance d'un certain «modernisme» pédagogique, tels que la concertation ou l'innovation, sont dépourvus d'effets significatifs en matière d'efficacité.

Notons aussi que certains facteurs d'organisation ou certains choix pédagogiques peuvent n'avoir aucun effet notable sur l'efficacité mais être importants en termes d'équité, accroissant ou réduisant significativement les écarts entre les élèves. C'est le cas des pratiques concernant la constitution des classes, dans lesquelles la responsabilité de l'établissement est directement engagée. Alors que les classes de niveau sont officiellement prohibées au collège depuis la réforme 
Haby (1975), seulement un quart des établissements constitueraient des classes relativement hétérogènes (Duru-Bellat \& Mingat, 1997). Or, en regroupant les élèves sur la base de leurs performances scolaires, on constitue aussi des classes homogènes sur le plan social et ethnique. Les collèges ont d'autant plus tendance à constituer des classes de niveau qu'ils accueillent un public défavorisé. Tout se passe comme s'ils tentaient ainsi d'enrayer la fuite des élèves des milieux les moins défavorisés en leur aménageant une classe «réservée» par le biais d'options particulières. Aucune option n'a à cet égard de vertus spécifiques, il peut s'agir du sport, de la musique ou de tout autre chose, l'essentiel est que l'accès en soit strictement contrôlé et régi par des règles informelles qui ne sont accessibles qu'aux «initiés» comme le montrent les analyses de Payet (1995) sur les collèges de banlieue. Selon les contextes, chaque établissement peut décider, avec la complicité de certaines familles, de mettre en place des classes spécifiques, dans une relative opacité. Ceci n'est pas anodin: bien qu'elles soient toujours formées dans l'intérêt des élèves, les classes destinées aux plus faibles d'entre eux les enfoncent encore plus, tant elles constituent souvent un milieu d'apprentissage très défavorable. Il est vrai que les élèves les plus forts "gagnent» à être regroupés entre eux, mais leur bénéfice est bien moindre que la perte des plus faibles. Au total, les classes de niveau accroissent les écarts de performances et d'attitudes scolaires entre les élèves. Au-delà des différences tenant aux publics des établissements, il faut souligner que les collèges créent eux-mêmes, par leurs pratiques de constitution de classes, une certaine ségrégation scolaire et sociale tout aussi importante que celle qui est induite par le jeu de la diversité des quartiers et de la carte scolaire.

Peut-on pour autant tirer de ces recherches un portrait type de l'établissement efficace (et équitable)? Rien n'est moins sûr, du fait des effets d'interaction qui existent en la matière; en d'autres termes, les modes de fonctionnement qui s'avèrent positifs en moyenne, pour l'ensemble des élèves, ne le sont pas forcément pour tous, pour les faibles comme pour les forts, les plus favorisés comme les plus défavorisés... Les faits confortent, avec nuances, cette thèse: Grisay (1997) montre qu'en milieu défavorisé, l'obsession de la discipline ou la raideur de fortes exigences peuvent être contre-productives (alors qu'elles s'avèrent propices aux progressions dans les collèges plus favorisés), tandis que la chaleur des relations, l'encouragement à la réussite de tous, le soutien parental y seraient plus importants. Mais pour autant, on ne trouve pas d'établissement qui serait efficace pour les élèves favorisés et inefficace pour les plus défavorisés. Les voies de l'efficacité sont donc multiples: aucun modèle canonique ne se dégage et les établissements performants sont loin d'offrir tous le même visage.

\section{Effet établissement et/ou effet mâ̂tre?}

Au total, nombre de résultats peuvent apparaître décevants pour les militants pédagogiques: ni le travail en équipe, ni la mise en place d'actions innovantes ou 
l'implication des enseignants dans le projet d'établissement n'apparaissent significativement liés à l'efficacité de l'établissement. Ces constats s'expliquent sans doute par le fait que la variance des pratiques déclarées par les enseignants d'un même collège est aussi grande que celle des enseignants de l'ensemble du territoire. Un constat identique est fait par Bressoux (1995): au sein d'une même école (primaire), les enseignants déclarent des pratiques pédagogiques très différentes qu'il s'agisse de leur style pédagogique (plus ou moins différenciateur), ou de la constitution de groupes de niveau; de même, les représentations des maîtres (concernant leur rôle quant à la réussite des élèves) ou encore leurs attentes sont extrêmement peu homogènes à l'intérieur d'une école. Il y a en fait beaucoup plus de différences au sein des écoles qu'il n'y en a d'une école à l'autre, ce qui rend évidemment peu probable l'existence d'effets école massifs. La recherche dément donc, dans le contexte français, la notion d'identité forte ou de climat spécifique à l'école ou à l'établissement.

Certaines analyses relevant de la sociologie des organisations éclairent ce type de résultats notamment ceux qui mettent en avant la notion de «bureaucratie professionnelle», dont les écoles seraient assez représentatives. Elles sont en effet caractérisées par un pouvoir largement décentralisé, une quasi-absence de ligne hiérarchique, l'existence de professionnels dûment qualifiés, effectuant un travail complexe dont il est difficile de standardiser les procédures et d'évaluer les résultats. Dans ce contexte, les enseignants ont une latitude considérable et peuvent de fait fonctionner à l'instar d'une profession libérale. On doit donc s'attendre à observer des «effets maître» bien plus sensibles que les effets établissement.

Avant d'aborder ce second champ de recherche, notons que cette relative faiblesse des effets école et, en positif, cette autonomie de l'enseignant sont sans doute plus marquées en France que dans certains pays voisins. Ainsi, les comparaisons menées par Broadfoot et Osborn (1987) entre la France et la Grande Bretagne font apparaître dans ce second pays davantage de coopération et d'échanges entre collègues. Cette différence procéderait elle-même de tout le contexte institutionnel; ainsi, jusqu'à une date récente, les enseignants britanniques avaient une certaine latitude dans la définition des programmes, ce qui exigeait à l'évidence des discussions locales; autre point, l'existence d'un directeur doté d'un réel pouvoir hiérarchique en Grande-Bretagne, élément vecteur lui aussi d'une certaine consistance du niveau école. D'ailleurs, dans les études du courant de la school effectiveness, le rôle spécifique du directeur apparaît toujours significatif, sauf précisément en France où ce rôle est moins affirmé, puisque le directeur n'est jamais que le "premier parmi ses pairs».

On ne saurait, pour conclure, retenir une image par trop sceptique de cette notion d'effet établissement: il faut souligner que la mobilisation au sein des établissements, si elle ne constitue pas une voie infaillible pour améliorer les choses, n'est pourtant pas sans portée. Ce que montrent les recherches, c'est que son contenu est essentiel: elle n'a d'effet sur les progressions des élèves que si elle porte sur le coeur même de la scolarité, sur ce qu'on fait en classe et non sur des 
aspects «périphériques» de la vie scolaire. C'est donc bien dans les classes, au plus près des pratiques, que se joue pour l'essentiel l'efficacité. Avec comme conséquence, pour le chercheur, qu'il est nécessaire d'articuler les analyses de l'effet établissement avec celles des effets maître.

Dans les pays anglo-saxons, l'analyse des effets des caractéristiques et des pratiques des maîtres sur les acquisitions des élèves est une longue et riche tradition (pour une synthèse, cf. Bressoux, 1994). Ils concordent pour souligner que, si les caractéristiques personnelles des enfants restent les facteurs les plus prédictifs de la réussite sur l'ensemble de la carrière scolaire, les effets maître s'avèrent très importants à chacun des niveaux successifs: des élèves comparables y progressent plus ou moins selon le maître avec lequel ils sont scolarisés. Ce courant de recherche postule, ce qui ne fait pas l'unanimité au sein de la recherche française, qu'il est possible et intéressant, même s'il s'agit à l'évidence d'une approche analytique abstraite, de faire la part, dans les inégalités d'acquis (ou d'attitudes) entre élèves, entre ce qui relève de leur expérience non scolaire, et ce qui est dû spécifiquement au fonctionnement de l'institution, en particulier aux pratiques pédagogiques des enseignants.

Depuis peu, ces effets mâ̂tre ont été étudiés en France, tant en primaire (Mingat, 1984, 1991; Serra \& Thaurel-Richard, 1994; Bressoux, 1995) que dans le secondaire (Felouzis, 1997). Ils s'avèrent à la fois marqués et durables. Au cours de la première année du primaire, les progressions sont plus affectées par le maître de l'enfant que par son origine sociale. Cet effet maître, qui peut aller jusqu'à expliquer, selon les recherches, entre 10 et $15 \%$ de la variance des progressions une année donnée, est en outre durable, les élèves ayant eu alors un maître «efficace» progressant mieux (encore) deux années plus tard. Enfin, les élèves faibles sont plus sensibles aux effets maître; autrement dit, les maîtres efficaces se singularisent par leur capacité à faire progresser davantage ce type d'élèves, s'avérant par là plus égalisateurs.

\section{L'efficacité des pratiques pédagogiques}

Dans l'explication de ces différences d'efficacité entre les maîtres, leurs caractéristiques personnelles (sexe, âge, formation) comptent très peu, à l'exception de l'ancienneté, associée, jusqu'à un optimum situé autour de 13-15 ans, à une meilleure efficacité. Par contre, la gestion du temps constitue un paramètre crucial: selon les maîtres, le temps consacré aux apprentissages, et à telle ou telle discipline varie sensiblement; par exemple, en première année de primaire, le temps alloué au français peut varier du simple au double, ce qui une fois de plus atteste son autonomie, malgré l'existence de règles nationales (Suchaut, 1996; pour les pays voisins, cf. Crahay, 2000). En l'occurrence, le maître efficace (à cet égard) est celui qui parvient à maximiser le temps pendant lequel les élèves sont actifs en termes d'apprentissage. Ceci passe par une certaine "gestion» du groupe 
classe: les maîtres efficaces savent prendre en charge tous les élèves, passer d'une chose à l'autre sans rupture, maintenir un rythme continu, proposer aux élèves des activités adaptées à leur niveau, maximisant ainsi leurs occasions d'apprendre.

Les effets d'attente sont également décisifs. Le maitre est plus efficace s'il est convaincu que ses élèves peuvent progresser: ceux-ci, exposés à des interactions pédagogiques plus stimulantes, s'efforceraient de répondre aux attentes des maitres; de fait, les élèves dont on attend beaucoup progressent plus que ceux dont on attend peu, conformément à ce qu'on appelle l'effet Pygmalion. Les enseignants ont tendance à "ancrer» leurs attentes sur des critères comme le sexe, l'appartenance sociale ou ethnique, appréhendée par l'apparence physique, la façon de s'habiller ou de s'exprimer, ou encore, et de plus en plus au fur et à mesure du déroulement du cursus, sur des indicateurs de valeur scolaire, résultats antérieurs, filières ou groupes de niveau. Toujours est-il que ces attentes, fondées sur les représentations, inévitablement stéréotypées, des élèves censés réussir ou échouer, participent à la reproduction des régularités statistiques sur lesquelles elles se fondent, puisqu'elles amènent à stimuler plus les élèves déjà "promis» à une meilleure réussite, et réciproquement. Dans cette perspective, relevant de la théorie de l'étiquetage, la réussite scolaire ne s'explique pas avant tout par les compétences intrinsèques des élèves mais par le jeu des attentes des maîtres, qui tendent à fonctionner comme des prophéties auto-réalisatrices.

On peut à cet égard noter qu'une partie de ces inégalités sociales créées par l'école provient paradoxalement de la diffusion des connaissances produites par la sociologie de l'éducation. Ainsi, les enseignants ont-ils tendance à sous-estimer le niveau des élèves de milieu populaire (Meuret \& Alluin, 1998), développant donc des attentes moins exigeantes à leur encontre, laissant se développer davantage de problèmes de discipline qui, même mineurs, obèrent les chances d'apprendre. Même si l'on comprend aisément que les maîtres partagent une représentation de l'étiologie des difficultés des élèves qui les protège, avec la thèse du handicap socioculturel tout puissant, le problème est que ces représentations ne sont pas sans affecter les pratiques pédagogiques. Etre convaincu que les enfants de milieu populaire abordent le collège avec de grandes difficultés débouche sur des pratiques qui les entérinent, en s'adaptant à ces difficultés et en le faisant sentir aux élèves.

Cela dit, à l'instar des effets établissement, il n'existe pas de pratique efficace dans l'absolu, quel que soit le contexte d'enseignement. Si tous les travaux soulignent l'importance du temps de travail actif de l'élève et des attentes des maîtres, la plupart des relations dégagées jouent en interaction avec le type de public d'élèves concerné (Bressoux, 1994). Par exemple, un enseignement directif semble plus efficace aux premiers stades de la scolarité, alors qu'aux niveaux plus élevés, c'est un enseignement moins directif qui s'avère plus productif. Autre exemple, avec les élèves de milieu favorisé, les maîtres les plus efficaces sont très exigeants, maintiennent un haut niveau de stimulation, critiquent volontiers, 
alors qu'avec des élèves de milieu défavorisé (qui ont en général d'eux-mêmes une image plus négative), il est plus efficace d'encourager, d'essayer de motiver, de minimiser les critiques, etc... Au-delà des difficultés pédagogiques concrètes que ce type de résultats laisse escompter, il est clair que l'évaluation d'une pratique d'enseignement ne peut se limiter à la comparaison des niveaux moyens, mais doit également prendre en compte la dispersion des résultats et leur corrélation avec le niveau initial.

Une question cruciale est bien en effet de savoir si certaines pratiques sont, plus que d'autres, à même d'atténuer ou au contraire de creuser les inégalités (notamment sociales) de résultats scolaires. Les travaux évaluant précisément les effets des pratiques pédagogiques ne sont pas légion en France, et les résultats sont contrastés. Ainsi, Isambert-Jamati (1990) montre, à propos de l'enseignement du français en lycée, qu'il ne suffit pas de rendre l'enseignement du français moins «littéraire» pour qu'il devienne plus démocratique. A cet égard, le caractère plus ou moins «visible» de la pédagogie (selon l'expression de Bernstein), c'est-à-dire le fait d'expliciter son mode de transmission, ses attentes et ses références, est sans doute beaucoup plus déterminant, ce que confirment les travaux récents de Barrère (1997) sur les lycéens. Néanmoins, une recherche récente au niveau collège (Tupin, 1996) montre que prendre en compte les acquis culturels des élèves de milieu populaire (culture télévisuelle notamment) peut leur permettre de réussir significativement mieux, et contribuer ainsi à réduire les écarts sociaux de réussite en français.

D'autres pratiques, présentées comme innovantes, censées représenter un progrès (par exemple, l'enseignement des langues par des méthodes audio-orales, ou leur enseignement précoce), accentuent en réalité les inégalités de réussite. Ceci vaut également pour certaines pratiques traduisant un souci de différenciation de l'enseignement, telle que la constitution de classes de niveaux, comme nous l'avons évoqué précédemment. Mais les progressions des élèves peuvent s'avérer meilleures et moins inégales si le souci d'adaptation au niveau de l'élève se traduit par l'organisation de groupes homogènes flexibles (dans la perspective de la pédagogie de maîtrise, cf Crahay, 2000), ou encore, plus simplement, par des annotations détaillées sur les copies ou des conseils personnalisés (Thaurel-Richard et Verdon, 1997; Schmitt-Roland \& Thaurel-Richard, 1996). De même, au niveau du lycée, Felouzis (1997) montre que les professeurs les plus efficaces sont ceux qui ont une vision réaliste du niveau des élèves et s'efforcent d'y adapter leurs pratiques sans pour autant baisser leurs exigences.

Toujours est-il que l'importance cruciale des attentes des enseignants amène à se poser la question de savoir comment faire en sorte que les enseignants «croient» davantage dans les possibilités de leurs élèves, et, de manière jointe, se croient eux-mêmes capables de les faire effectivement progresser. La littérature sur la mobilisation professionnelle des enseignants est à cet égard éclairante. Elle montre qu'en laissant entrevoir la possibilité de mieux maitriser et de transformer l'exercice quotidien du métier, la mobilisation des communautés éduca- 
tives fait émerger des identités professionnelles plus réfléchies et plus positives 5 , ces dernières étant porteuses à terme d'une meilleure efficacité. La mobilisation s'enracine dans le travail d'équipe en fonction de réseaux structurés par les affinités personnelles bien plus que par des choix idéologiques. En effet, seules des relations de confiance permettent aux enseignants de dépasser une conception individualiste du travail et la peur de s'exposer au regard des autres. C'est essentiellement en ce qu'elle brise l'isolement des enseignants et le face à face exclusif avec les élèves que la mise en œuvre concertée de pratiques objectivement banales peut remodeler profondément le métier. On sait que l'isolement traditionnel des professeurs les conduit à s'appuyer davantage sur la vision du rôle d'enseignant acquise alors qu'ils étaient élèves, plutôt que sur des ressources extérieures. En arrachant le métier à son caractère privé, en explicitant des modes de fonctionnement jusqu'alors aussi personnels qu' implicites, on s'achemine vers une définition publique et objectivée de la compétence professionnelle.

Mais si les professeurs ont tout à gagner à débattre sans tabou des difficultés rencontrées et de la manière concrète d'y faire face, il est certain que cela les contraint, comme les textes français les y enjoignent depuis 1989, à inscrire leur action dans le contexte collectif de l'établissement, perdant ainsi l'autonomie complète dont ils jouissaient jusqu'alors dans leurs classes. Une approche plus expérimentale de sa pratique devient nécessaire, soucieuse des effets de ce qu'on fait. La compétence des enseignants ne peut plus alors se définir exclusivement par la conformité au rôle, par la transmission de connaissances fondée sur la maîtrise de contenus disciplinaires; elle doit se faire plus attentive aux apprentissages des élèves et à leurs ressorts. L'effet maître viendra alors signer la responsabilité de l'enseignant, la valeur de sa compétence professionnelle.

\section{Des questions de recherches ouvertes, aux débouchés politiques controversés}

Tout ce qui précède laisse à penser que l'établissement (ou le maître) fréquenté joue sur le devenir scolaire de l'élève. Mais il faut souligner que tous ces résultats sont l'objet, dans la littérature anglo-saxonne, de débats passionnés quant à leur fiabilité scientifique et leur signification politique. On ne peut guère imaginer de tels débats en France, tant les méthodes suivies par les chercheurs restent frustes et uniformes, tant aussi certaines questions restent largement tabou. Parmi les controverses anglo-saxonnes, évoquons celle portant sur l'ampleur et la signification des effets de contexte. Il est clair que les effets établissement ou les effets maître sont sensibles aux modèles d'analyse retenus: selon que l'on met en oeuvre des modèles multiniveaux ou des régressions ordinaires (Bressoux, 1995), ils seront plus ou moins importants.

Ceci n'est pas sans incidence en termes politiques comme le soulignent des chercheurs britanniques (Gibson \& Asthana, 1999): à leurs yeux, les approches 
en termes de valeur ajoutée, qui mettent en exergue le rôle du contexte, amènent par contrecoup à négliger le rôle des handicaps sociaux des élèves, et les contraintes qu'ils font peser sur les écoles, dont attestent très clairement les corrélations entre réussite et type de public (ou tous les indicateurs plus frustes comme les taux bruts). Or est-on vraiment certain que les facteurs d'organisation propres à l'établissement, que ses choix pédagogiques, bref tout ce qu'il est en son pouvoir de faire, pèse de manière significative quel que soit le public qu'il accueille? La réponse du courant de recherche dit de la school effectiveness est positive. Mais elle ne fait pas l'unanimité. Certains soutiennent au contraire qu'il est vain de prétendre dégager, par des analyses de type valeur ajoutée des facteurs d'efficacité qui vaudraient dans tous les cas et qu'il serait donc de la responsabilité des écoles de mettre en place (Thrupp, 1995 et 1999; Gibson \& Asthana, 1999). La plupart des recherches sur les effets établissement montrent qu'effectivement la composition sociale du public joue sur le climat que l'on parvient à mettre en place dans l'établissement, la couverture des programmes, les exigences envers les élèves. De manière régulière, les écoles qui accueillent un public favorisé se montrent plus efficaces; il s'avère toujours plus facile d'être efficace quand les élèves "apportent avec eux" un certain nombre d'atouts, qu'il s'agisse d'acquis initiaux mais aussi d'attitudes plus ou moins favorables à l'exercice tranquille du métier d'élève. Dès lors, comme le dit Thrupp, beaucoup de facteurs d'efficacité sont de fait school based et non school caused.

Les incidences politiques de cette question de recherche sont clairs: si les facteurs d'efficacité sont indissociables de la composition du public d'élèves, alors il est tendancieux de les présenter comme des pistes d'action que tous les établissements pourraient suivre. En particulier, les écoles populaires vont être désignées comme coupables de peiner à s'améliorer... Néanmoins, les analyses de chercheurs comme Grisay (1997) montrent que ces écoles peuvent aussi s'avérer efficaces, sans doute par les voies différentes de celles que les écoles privilégiées trouvent en quelque sorte (et plus souvent) dans leur berceau. Au-delà de ces deux perspectives - privilégiant soit les marges d'action de l'école, soit ses contraintes -, il y a un équilibre à trouver, sachant qu'au niveau de la recherche, on gagne évidemment à la multiplicité des points de vue.

Toujours est-il que si le school mix - la composition du public d'élèves - reste le facteur le plus décisif en termes d'efficacité d'une école, alors les comportements de choix des familles de milieu aisé deviennent des plus rationnels (puisqu'on progresserait davantage dans une école "chic», à la limite indépendamment de tous les autres facteurs). Le politique soucieux de ne pas laisser dériver les écoles populaires, ce qui entraînerait des cô̂ts sociaux importants, aura à se montrer très offensif, pour contrer ces comportements de choix d'école, conformes aux intérêts individuels: il peut le faire en pratiquant une forte discrimination positive, en s'acharnant à rendre plus attractives les écoles populaires de plus en plus délaissées, pour que les familles les mieux informées n'aient plus de «bonnes raisons» de les fuir. La mixité sociale des écoles peut être un objectif 
politique en soi, et les recherches peuvent la justifier en montrant que les écoles ghettos creusent les écarts. Mais il reste la question essentielle des voies (toutes politiques) permettant d'en convaincre les familles et les enseignants, sans se contenter d'édicter des règles que les mieux informés, les parents enseignants en premier lieu, enfreignent sans problème. Dans ce conflit classique entre intérêt général et intérêts particuliers, l'enjeu est tout simplement l'existence même d'un système éducatif.

\section{Notes}

1 On sait en effet que par leur notation, les établissements composent avec leur public: celleci est d'autant plus indulgente que l'établissement a affaire à un public faible, l'inverse étant vrai avec un public de bon niveau scolaire (cf. Duru-Bellat \& Mingat, 1988).

2 Il est clair qu'en l'absence de travail comparatif sur un nombre important d'établissements, l'appréhension ethnographique de l'identité de l'établissement ne peut être que très floue et souvent trompeuse, pouvant conduire à considérer comme une spécificité de l'établissement ce qui relève de phénomènes contextuels très généraux.

3 Au niveau primaire comme dans le secondaire, la relative homogénéité française est sans doute une conséquence de la centralisation.

4 L'inégalité des ressources matérielles entre établissements ou entre sites a été longtemps sous-estimée en France, du fait de la centralisation. Il faut attendre les années 90 pour que tant des rapports officiels que des mouvements sociaux attirent l'attention sur les inégalités de dotation en personnels ou plus largement en moyens, selon les départements par exemple.

5 Sur les modifications du métier induites par la mobilisation et plus largement les publics difficiles cf. Bautier (dir.), 1995; Kherroubi, Peignard, et Robert, 1997 et 1998. Pour une synthèse, cf. Chatel, Rochex et Roger, 1996.

\section{Références}

Barrère, A. (1997). Les lycéens au travail. Paris: Presses Universitaires de France.

Bautier, E. (Ed.) (1995). Travailler en banlieue. Paris: L'Harmattan.

Bressoux, P. (1994). Les recherches sur les effets-école et les effets-maîtres. Revue Française de Pédagogie, 108, 91-137.

Bressoux, P. (1995). Les effets du contexte scolaire sur les acquisitions des élèves: effet-école et effets-classes en lecture. Revue Française de Sociologie, 36 (2), 273-294.

Broadfoot, P. \& Osborn, M. (1987). Teachers'Conceptions of their Professional Responsibility: some international comparisons. Comparative Education, 23 (3), 287-301.

Chatel, E., Rochex, J.-Y. \& Roger, J.-L. (1996). Transformations du second degré et métier d'enseignant. In C. Grémion \& R. Fraisse (Ed.), Le service public en recherche. Quelle modernisation? (pp. 329-338). Paris: La Documentation Française.

Coleman, J.S. (1966). Equality of Educational Opportunity. Washington D.C.: Government Printing Office.

Cousin, O. (1998). L'efficacité des collèges. Sociologie de l'effet établissement. Paris: Presses Universitaires de France.

Crahay, M. (2000). L'école peut-elle être juste et efficace? Bruxelles: De Boeck.

Derouet, J.-L. (1992). Ecole et justice. Paris: Métaillé.

Duru-Bellat, M. \& Mingat, A. (1988). Le déroulement de la scolarité au collège: le contexte «fait des différences». Revue Française de Sociologie, 29, 649-666. 
Duru-Bellat, M. \& Mingat A. (1997). La constitution de classes de niveau par les collèges: les effets pervers d'une pratique à visée égalisatrice. Revue Française de Sociologie, 38, 759-790.

Felouzis, G. (1997). L'efficacité des enseignants. Paris: Presses Universitaires de France.

Gibson, A. \& Asthana, S. (1999). Schools, Markets and Equity; access to secondary education in England and Wales. American Educational Research Association Annual Meeting, Montréal, 21/04/99.

Grisay, A. (1993). Le fonctionnement des collèges et ses effets sur les élèves de 6e et 5e. Les Dossiers d'Education et Formations, 32.

Grisay, A. (1997). L'évolution des acquis cognitifs et socio-affectifs des élèves au cours de années de collège. Les Dossiers d'Education et Formations, 88.

Isambert-Jamati, V. (1990). Les savoirs scolaires. Paris: Ed.Universitaires.

Kherroubi, M., Peignard, E. \& Robert, A. (1997). Des enseignants et des établissements mobilisés. Carrefour de l'Education, 3, 60-75 (1ère partie).

Kherroubi, M., Peignard, E. \& Robert, A. (1998). Des enseignants et des établissements mobilisés. Carrefour de l'Education, 6, 42-63 (2ème partie).

Meuret, D. (1995). Distribution sociale des facteurs d'efficacité des collèges. In J.-M. Besse (et al.) (Ed.), Ecole efficace: de l'école primaire à l'Université (pp. 81-91). Paris: Colin.

Meuret, D. \& Marivain, T. (1997). Inégalités de bien-être au collège. Les Dossiers d'Education et Formations, 89.

Meuret, D. \& Alluin, F. (1998). La perception des inégalités entre élèves par les enseignants du second degré. Education et Formation, 53, 67-78.

Mingat, A. (1984). Les acquisitions scolaires de l'élève au CP: les origines des différences. Revue Française de Pédagogie, 69, 49-63.

Mingat, A. (1991). Expliquer la variété des acquisitions au CP: les rôles de l'enfant, la famille et l'école. Revue Française de Pédagogie, 95, 47-63.

Payet, J.-P. (1995). Collèges de banlieue. Paris: Méridiens-Klincksieck.

Serra, N. \& Thaurel-Richard, M. (1994). Acquisitions des élèves au CE2 et pratiques pédagogiques. Revue Française de Pédagogie, 107, 43-62.

Schmitt-Roland, S. \& Thaurel-Richard, M. (1996). Pratiques pédagogiques de l'enseignement du Français en Gème et progrès des élèves. Les Dossiers d'Education et Formations, 87.

Suchaut, B. (1996). La gestion du temps à l'école maternelle: diversité des pratiques et effet sur les acquisitions des élèves. L'Année de la recherche en Education, 123-153.

Thaurel-Richard, M. \& Verdon, R. (1997). Pratiques pédagogiques de l'enseignement des mathématiques en Gème et progrès des élèves. Les Dossiers d'Education et Formations, 84.

Thrupp, M. (1995). The "school mix» Effect: the history of an enduring problem in educational research, policy and practice. British Journal of Sociology of Education, 16 (2), 183203.

Thrupp, M. (1997). How School Mix Shapes School Processes: A Comparison of New Zealand Schools. New Zealand Journal of Educational Studies, 32 (1), 53-82.

Thrupp, M. (1999). Schools Making a Difference. Let's be Realistic! Buckingham: Open University Press.

Tupin, F. (1996). Espace d'action des enseignants et démocratisation: l'exemple de l'enseignement de la narration écrite au collège. Revue Française de Pédagogie, 115, 67-77. 


\section{Lehrereffekte, Schuleffekte: Welche Verantwortung trägt die Schule?}

\section{Zusammenfassung}

In Frankreich wird die Heterogenität der Bildungsbedingungen und der schulischen Kontexte als einer der wichtigen Aspekte der Krise der Schule angesehen. Aber gibt es da nur die Übertragung der Segregation der sozialen Verhältnisse auf die Schule? Oder sollte man zugestehen, dass die Schule selbst an der Definition unterschiedlichster Bildungsbedingungen beteiligt ist? Der folgende Text präsentiert eine aktuelle Synthese französischer Forschungsresultate zur Frage der Kontexteffekte, insbesondere der Lehrereffekte und der Schuleffekte. Dabei liegt das Gewicht auf den methodologischen Problemen, die sich stellen, auf deren Nuancen und deren Komplexität (die Resultate stehen ja selber wiederum in einem bestimmten Kontext), und es wird auf die Notwendigkeit hingewiesen, Wirkung und Gerechtigkeit zu unterscheiden. Zudem wird auf die Kontroversen, die zu den Forschungsresultaten geführt werden, eingegangen und zum Schluss auf die Frage nach den politischen Konsequenzen verwiesen: Geht es um die Mobilisierung der Lehrerschaft, um die "Kontrolle" der elterlichen Ansprüche oder - grundlegender - um die Möglichkeit der Schule, allen ein pädagogisches Umfeld von gleicher Qualität anzubieten?

\section{Effetto insegnanti, effetto istituto scolastico: quali le responsabilità della scuola?}

\section{Riassunto}

In Francia la differenza tra le varie situazioni educative e i contesti scolastici viene volentieri considerata come uno degli aspetti importanti della crisi della scuola. Ma trattasi solo della traduzione nella realtà scolastica della segregazione esistente a livello sociale? Oppure occorre ammettere che la scuola stessa contribuisce alla definizione di tali diversità? Questo contributo presenta una sintesi attualizzata delle ricerche francesi dedicate agli effetti del contesto - dell'insegnante e dell'istituto scolastico - e insiste sui problemi metodologici che ne derivano, sulla specificità e complessità dei dati (a loro volta contestualizzati), sulla necessità di distinguere tra efficacia ed equità. Sottolinea pure le controversie nonché la valenza politica legate alla questione, sia che si tratti della mobilizzazione degli insegnanti, del controllo delle richieste dei genitori o, più fondamentalmente, della capacità dell'istituzione scolastica di offrire a tutti un ambito educativo di eguale qualità. 


\section{Teacher effects, school effects: what is the responsibility of schools?}

\section{Summary}

In France, the heterogeneity of the conditions of education and of school's contexts is viewed as one of the most important aspects of the crisis of schools. But is there only the transfer of the segregation of social circumstances onto school? Or should one admit that school itself is involved with the definition of various conditions of education? The following text presents a current synthesis of French research results regarding the question of effects of context, especially effects of teachers and schools. Thereby the weight lies on the methodologistic problems that appear, on their nuances and on their complexity (the results themselves occur in a fixed context), and the necessity is indicated to differentiate between its effect and fairness. In addition, the contribution delves into the controversies that led to the research results and concludes by pointing to a question of political consequences: is it about the mobilisation of the teaching body, the control of parental demands, or even more fundamentally, the feasibility of schools to offer everybody a pedagogic environment of equal quality? 
T h e m a 